\title{
Year of Onset
}

National Cancer Institute

\section{Source}

National Cancer Institute. Year of Onset. NCI Thesaurus. Code C99800.

The year in which an event began. 